\title{
Influence of transverse mode on retinal spot size and retinal injury effect: A theoretical analysis on 532-nm laser
}

\author{
Jia-Rui Wang*, , Guang-Yuan Yu ${ }^{\dagger, \boldsymbol{\pi}}$, Zai-Fu Yang**, \\ Lu-Guang Jiao*, Hong-Xia Chen ${ }^{\dagger}$ and Xian-Biao Zou ${ }^{\dagger}, \S$ \\ *Department of Laser and Electromagnetic Biology \\ Beijing Institute of Radiation Medicine, Beijing 100850, P. R. China \\ ${ }^{\dagger}$ Department of Dermatology, First Affiliated Hospital \\ of PLA General Hospital, Beijing 100048, P. R. China \\ †lasermp3@nic.bmi.ac.cn \\ §xbzou@126.com
}

Received 31 May 2013

Accepted 20 October 2013

Published 16 December 2013

\begin{abstract}
The fundamental transverse mode $\left(\mathrm{TEM}_{00}\right)$ is preferable for experimental and theoretical study on the laser-induced retinal injury effect, for it can produce the minimal retinal image and establish the most strict laser safety standards. But actually lasers with higher order mode were frequently used in both earlier and recent studies. Generally higher order mode leads to larger retinal spot size and so higher damage threshold, but there are few quantitative analyses on this problem. In this paper, a four-surface schematic eye model is established for human and macaque. The propagation of 532-nm laser in schematic eye is analyzed by the ABCD law of Gaussian optics. It is shown that retinal spot size increases with laser transverse mode order. For relative lower mode order, the retinal spot diameter will not exceed the minimum laser-induced retinal lesion (25 30 $\mu \mathrm{m}$ in diameter), and so has little effect on retinal damage threshold. While for higher order mode, the larger retinal spot requires more energy to induce injury and so the damage threshold increases. When beam divergence is lowered, the retinal spot size decreases correspondingly, so the effect of mode order can be compensated. The retinal spot size of macaque is slightly smaller than that of human and the ratio between them is independent of mode order. We conclude that the laser mode order has significant influence on retinal spot size but limited influence on the retinal injury effect.
\end{abstract}

Keywords: Laser transverse mode; four-surface schematic eye; retinal spot size; retinal injury effect.

IThese authors contribute equally to this work.

\$Corresponding author.

This is an Open Access article published by World Scientific Publishing Company. It is distributed under the terms of the Creative Commons Attribution 3.0 (CC-BY) License. Further distribution of this work is permitted, provided the original work is properly cited. 


\section{Introduction}

The retina of the eye is vulnerable to visible and near infrared laser which can propagate through the eye into a small spot. ${ }^{1-3}$ The injury threshold for exposure to a laser source varies with the irradiated area on the retina. Based on the study of thermal damage model by Zuclich et al., ${ }^{4}$ the dependence of retinal damage threshold on the retinal spot size varies with injury mechanism which is determined by exposure duration. For a pulse ranging from microseconds to seconds, the retina damage is induced by thermal effect, and the thresholds, in terms of total intraocular energy (TIE), are proportional to the retinal spot diameter. ${ }^{5-7}$ While for a pulse ranging from nanoseconds to microseconds, the damage is induced by nonthermal effects, such as microcavitation and plasma effect, and the thresholds are found to be a square dependence on the retinal spot diameter when it is larger than $80-100 \mu \mathrm{m} .{ }^{8,9}$ However, as the retinal spot diameter decreases to about $25-30 \mu \mathrm{m}$, the threshold will be independent of it, ${ }^{10}$ which is the basis for the establishment of laser safety standard.

According to the latest guidelines published by International Commission on Non-Ionizing Radiation Protection (ICNIRP), the dependence of maximum permissible exposure (MPE) on the retinal spot size (or source angular substance) varies with mechanism, ${ }^{11}$ as showed in Fig. 1. From above discussion, we can see that the retinal spot diameter is an important parameter in the research of retinal injury threshold.

Undoubtedly, experimental data achieved by using the $\mathrm{TEM}_{00}$ mode can establish the most strict

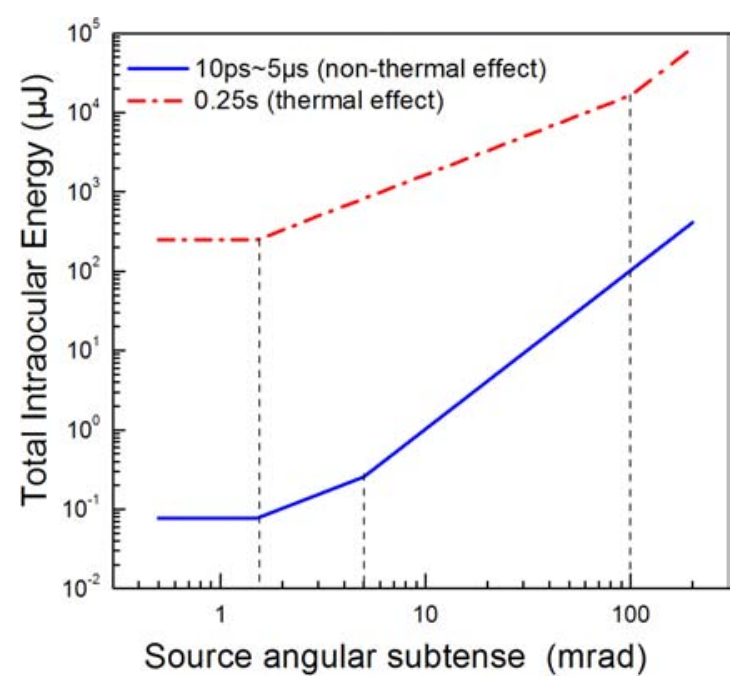

Fig. 1. Retinal spot size dependence of MPE corresponding to thermal and nonthermal damage mechanisms. laser safety standards for it can produce the minimal retinal image. But some of the lasers which was claimed to be in $\mathrm{TEM}_{00}$ mode by earlier investigators was presumed to be in multimode actually. The earlier thresholds were found to be generally larger than the recent values, which probably resulted from underestimation of the retinal spot size. ${ }^{12,13}$ Nowadays, with significant advances in laser technology, lots of lasers can export a beam with $\mathrm{TEM}_{00}$ mode. But the output power of the lasers with pure $\mathrm{TEM}_{00}$ mode might be too low to induce observable damage. So a question is raised that whether the higher order modes can be used in the experimental study on the laser injury thresholds. However, few quantitative analysis of the influence of higher order mode on the retinal injury effects have been performed in literature. In this paper, we aim to analyze the influence of laser transverse mode on the retinal spot size and retinal injury effect.

Theoretically, the laser spot diameter and intensity distribution on the retina can be inferred from the structure of the eye and the properties of incident laser beam. ${ }^{14-16}$ As a living dioptric system, the eye mainly consists of four media: cornea, aqueous, crystalline lens and vitreous. Different eye models for human and other animals have been established. ${ }^{17-24}$ Among these models, the classical simplified eye models with spherical surfaces and homogeneous refractive index are still widely used for paraxial ray calculations, ${ }^{14}$ such as the foursurface schematic eye model commonly accepted for human, macaque, rabbit and rat. ${ }^{21-24}$ Another issue which should be considered is how to characterize the propagation of higher order mode laser beam. According to the literature, the embedded Gaussian model, in which the higher order modes are treated as the $\mathrm{TEM}_{00}$ mode, is frequently used for its convenience. In our model, we also use the four-surface schematic eye model and the embedded Gaussian model to estimate the laser spot diameter on the retina. The laser wavelength in our calculation is $532 \mathrm{~nm}$ which is widely used for the study on the laser-retina injury effect.

\section{Methods}

\subsection{Embedded Gaussian model and $A B C D$ law}

The two main families of higher order transverse mode beams are Laguerre-Gaussian beam and 
Hermit-Gaussian beam. ${ }^{25,26}$ Most lasers export Laguerre-Gaussian beam with cylindrical symmetric irradiance pattern due to the circular resonance mirror. As shown in Fig. 2, the patterns of the higher order transverse modes, $\mathrm{TEM}_{p l}$, are described by two parameters which are radial order $p$ and azimuthal order $l$.

The "embedded Gaussian beam" is a convenient means by which the calculation can be simplified. ${ }^{27,29}$ The TEM $_{00}$ mode is embedded in it, as shown in Fig. 3. The beam parameters of $\mathrm{TEM}_{p l}$ mode as a function of the propagation distance from the waist position are expressed as follows:

beam radius at location $z$ :

$$
\begin{gathered}
W_{p l}(z)=w_{0} \sqrt{2 p+l+1} \sqrt{1+\left(\frac{z}{z_{0}}\right)^{2}}, \\
\text { waist radius: } W_{p l, z=0}=w_{0} \sqrt{2 p+l+1}, \\
\text { angle of divergence: } \theta_{p l}=\theta_{0} \sqrt{2 p+l+1}, \\
\text { beam propagation factor: } M^{2}=2 p+l+1 .
\end{gathered}
$$

In above equations, $\lambda$ is the laser wavelength, $z_{0}$ is the Rayleigh length, $w_{0}$ is the waist radius of the $\mathrm{TEM}_{00}$ mode (corresponding to the position where the local irradiance is $1 / e^{2}$ of the central maximum level $\left.^{27}\right), \theta_{0}$ is the divergence of the $\mathrm{TEM}_{00}$ mode,
$W_{p l}$ and $\theta_{p l}$ represent the waist radius and the divergence of higher order mode beam, respectively. ${ }^{27}$ The waist radius and the divergence of the higher order modes are $M$ times larger than that of the $\mathrm{TEM}_{00}$ mode. Consequently, the beam diameter of the higher order mode beam will always be $M$ times that of the $\mathrm{TEM}_{00}$ mode beam in any cross section. The complex parameter of the beam can be expressed as:

$$
\frac{1}{q_{p l}(z)}=\frac{1}{R_{p l}(z)}+i \frac{\lambda M^{2}}{\pi W_{p l}^{2}},
$$

where $R_{p l}(z)$ is the curvature radius of the wave front at distance $z$. In paraxial optical systems, the $\mathrm{ABCD}$ law is frequently used to describe the propagation of the Gaussian beam from one reference plane to another. After the beam propagates through an optical system, its complex parameter changes to:

$$
q_{p l_{2}}=\frac{A q_{p l_{1}}+B}{C q_{p l_{1}}+D} .
$$

Then the beam radius can be expressed as:

$$
W_{p l_{2}}^{2}=\frac{\lambda}{\pi \cdot \operatorname{Im}\left(1 / q_{p l_{2}}\right)},
$$

where Im denotes the imaginary part of a complex number.

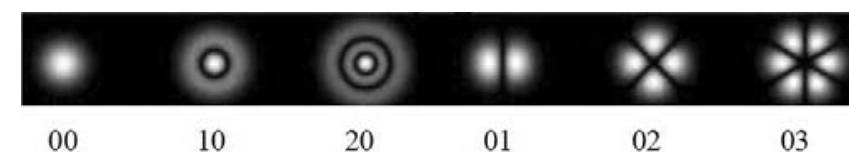

Fig. 2. Diagrams of intensity distributions of different Laguerre-Gaussian modes. The two numbers represent the radial order $p$ and azimuthal order $l$ in the sequence.

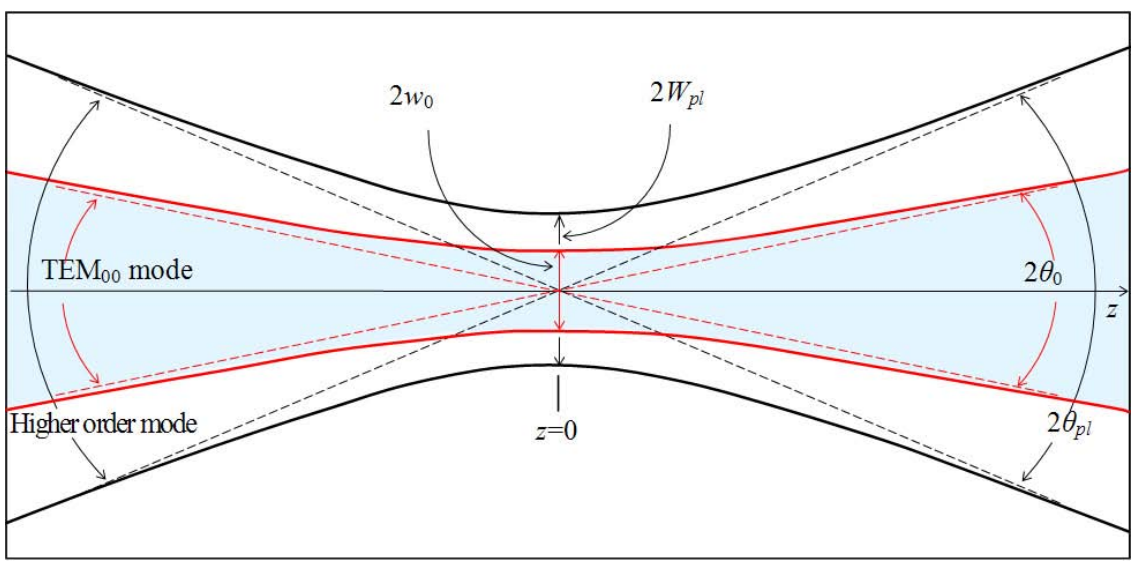

Fig. 3. The embedded Gaussian model. 


\subsection{Four-surface schematic eye model and retina localization}

The four-surface schematic eye model is established according to literatures. ${ }^{21,30}$ In the model, the following parameters are involved: the curvature of anterior and posterior surfaces of the cornea and lens, the thickness and the refractive index of the cornea, aqueous, lens and vitreous. All the interfaces in the eye model are ideal spherical surfaces. The details are shown in Fig. 4 and Table 1. The refractive indices of ocular media listed in Table 1 refer to the values at the wavelength of $532 \mathrm{~nm}$, which are calculated by using the values at $589 \mathrm{~nm}$ and assuming that the ocular media has the same dispersion with water. ${ }^{31}$ As the macaque is the most favored animal model for the retinal injury studies, we also listed its parameters for comparison with human.

By histology analysis, it is found that the laserinduced retinal damage is localized at the RPE layer. ${ }^{32}$ The RPE is a monolayer of cells containing melanin granules which is a strong absorber of optical radiation. The absorbed energy results in tissue heating and damage. ${ }^{33,34}$ In our model, the exact location of the retina (RPE layer) is set at the intraocular beam waist for paralleled $\mathrm{TEM}_{00}$ mode at $532 \mathrm{~nm}$, which is 16.57 and $12.93 \mathrm{~mm}$ post-lenticular in the human and macaque eye respectively, as shown in Table 1.

The transfer matrix for the ocular system can be obtained by multiplying eight individual matrices (four refractive media and four interfaces) in the opposite order of the ray propagation, as shown in Eq. (8).

$$
M=\left(\begin{array}{ll}
A & B \\
C & D
\end{array}\right)
$$

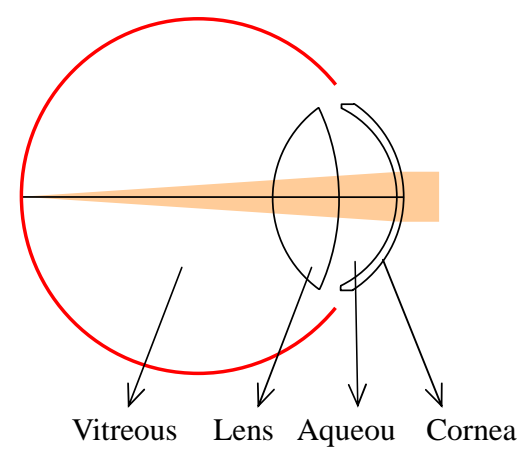

Fig. 4. The four-surface schematic eye model.
Table 1. Geometrical characteristics of schematic eye models.

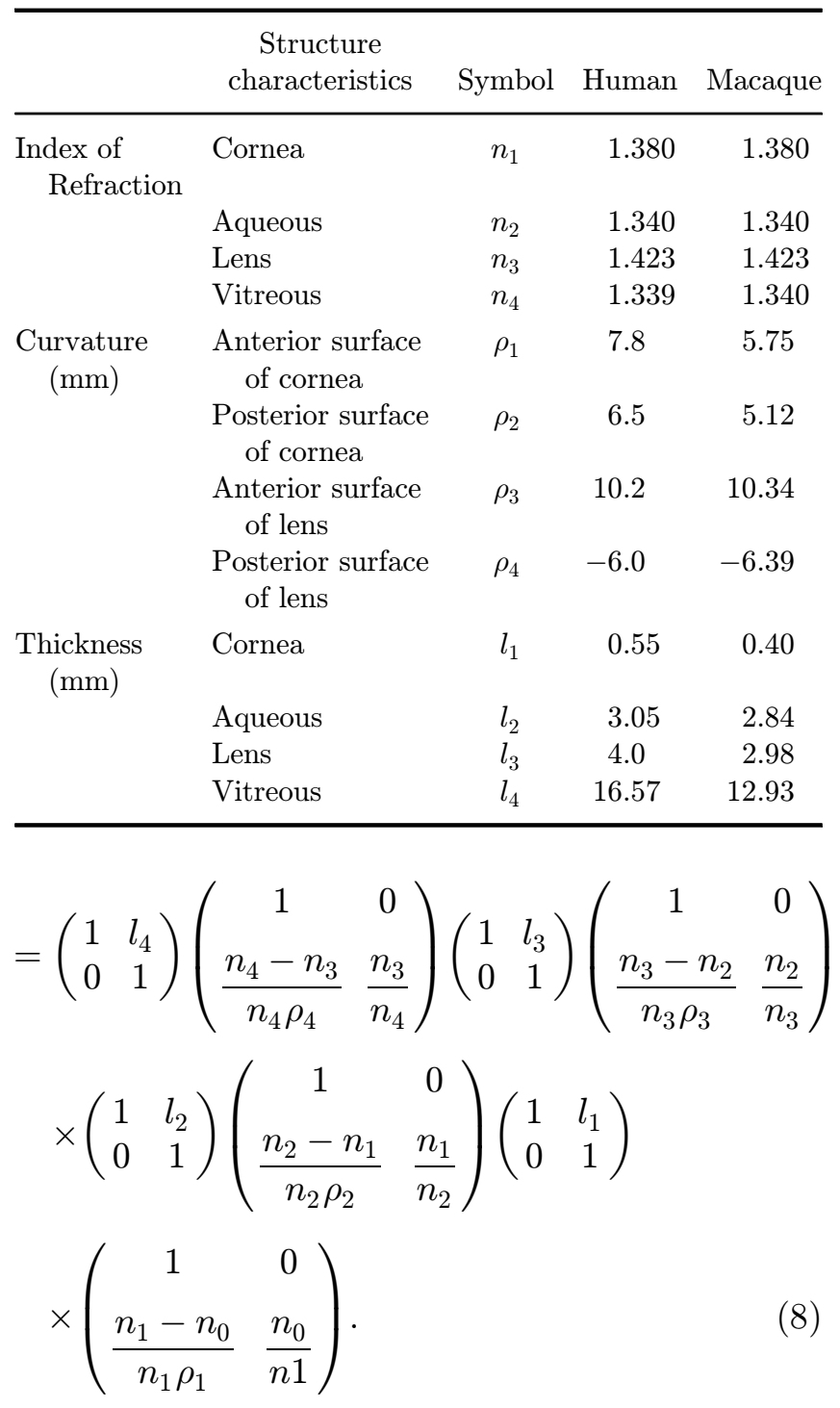

\section{Results}

\subsection{Intraocular propagation of different transverse order mode}

For the calculation of the retinal spot size, no diffraction effects or effect of the aperture on the beam are considered, i.e., the lens diameter is assumed to be infinite (or at least much larger than the beam diameter). ${ }^{35}$ In our model, the diameter of the pupil is set to $7 \mathrm{~mm}$. The laser parameters are as follows: wavelength - $532 \mathrm{~nm}$, distance from laser source to the anterior surface of cornea $-2.4 \mathrm{~m}$, beam divergence of the embedded $\mathrm{TEM}_{00}$ mode -0.2 and $0.5 \mathrm{mrad}$. The setting is a trade-off between the beam diameter and the pupil diameter. Figure 5 shows the 


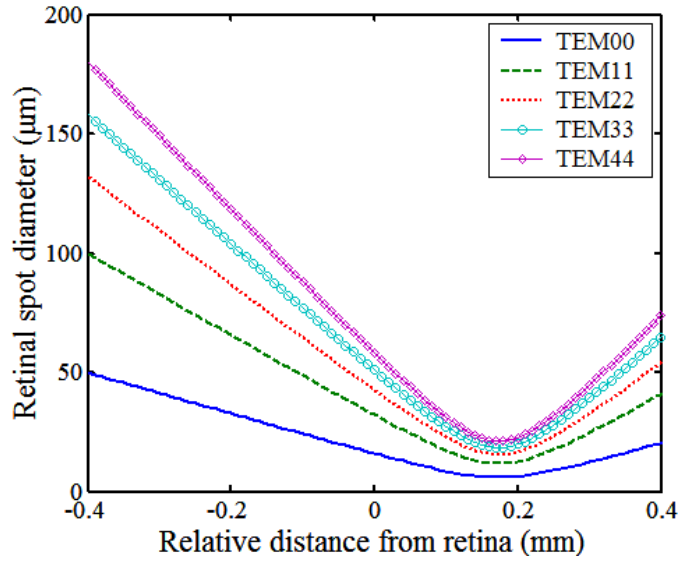

(a)

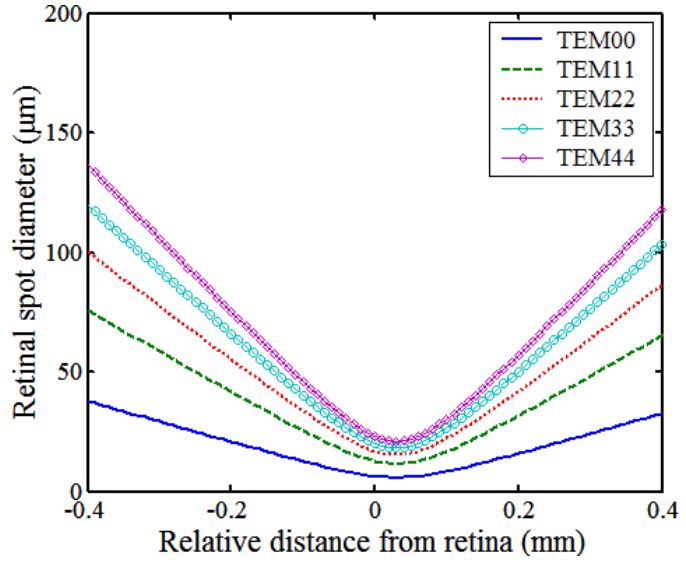

(b)

Fig. 5. Calculated beam diameter of different transverse modes focused by human eye under the conditions: wavelength - $532 \mathrm{~nm}$, distance from laser source to the anterior surface of cornea $-2.4 \mathrm{~m}$, beam divergence of the embedded TEM $\mathrm{mode}_{00}-0.2 \mathrm{mrad}$ (a), $0.5 \mathrm{mrad}$ (b).

intraocular propagation of different order modes in human eye. The retina is located at the zero position of the horizontal axis. As can be seen in Fig. 5, the beam waist of higher order mode locates at the same position as that of the $\mathrm{TEM}_{00}$ mode. The spot diameter on the retinal plane increases with the mode order. The calculated retinal spot diameter of $\mathrm{TEM}_{00}$ mode with the divergence of 0.2 and $0.5 \mathrm{mrad}$ is 6.4 and $16.1 \mu \mathrm{m}$, respectively.

\subsection{Influence of radial and azimuthal order on the retinal spot diameter}

Figure 6 shows the influence of radial and azimuthal order on the retinal spot diameter. The beam divergence of the embedded $\mathrm{TEM}_{00}$ mode is $0.2 \mathrm{mrad}$ and other laser parameters are same with Fig. 5. It can be seen that the effect of radial order is stronger than that of azimuthal order. The maximum retinal spot diameter corresponds to $\mathrm{TEM}_{44}$ mode, which is $23.1 \mu \mathrm{m}$ for human and $18.2 \mu \mathrm{m}$ for macaque.

\subsection{Influence of beam propagation factor on the retinal spot diameter}

The highest order of transverse mode involved in our calculation is $\mathrm{TEM}_{44}$ mode, which corresponds to the beam propagation factor 13. Figure 7(a) shows the dependence of the retinal spot diameter on beam propagation factor for human and

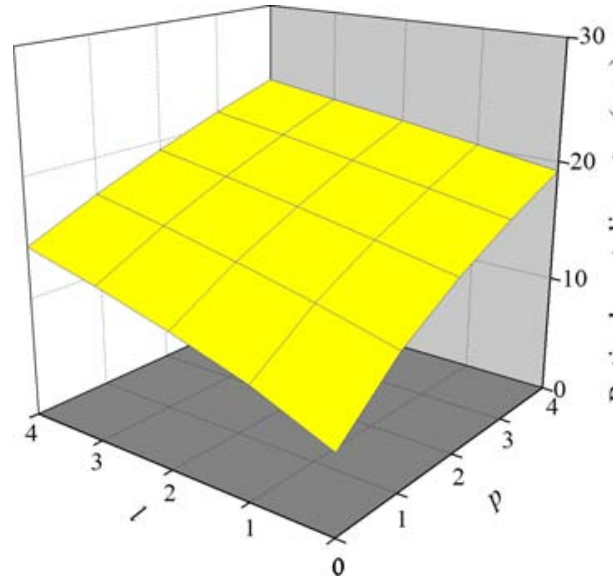

(a)

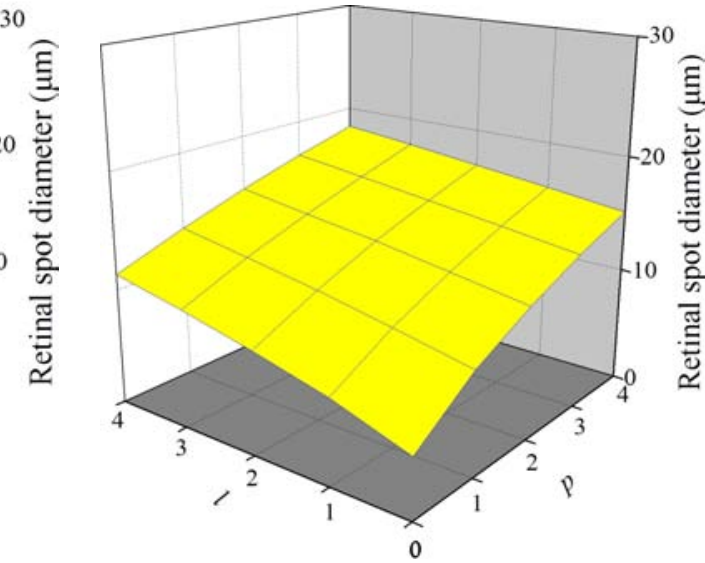

(b)

Fig. 6. Retinal spot diameter versus radial order $p$ and azimuthal order $l$ of transverse mode for human eye (a) and macaque eye (b). The beam divergence of the embedded $\mathrm{TEM}_{00}$ mode is $0.2 \mathrm{mrad}$, other parameters are same with Fig. 5 . 


\section{J.-R. Wang et al.}

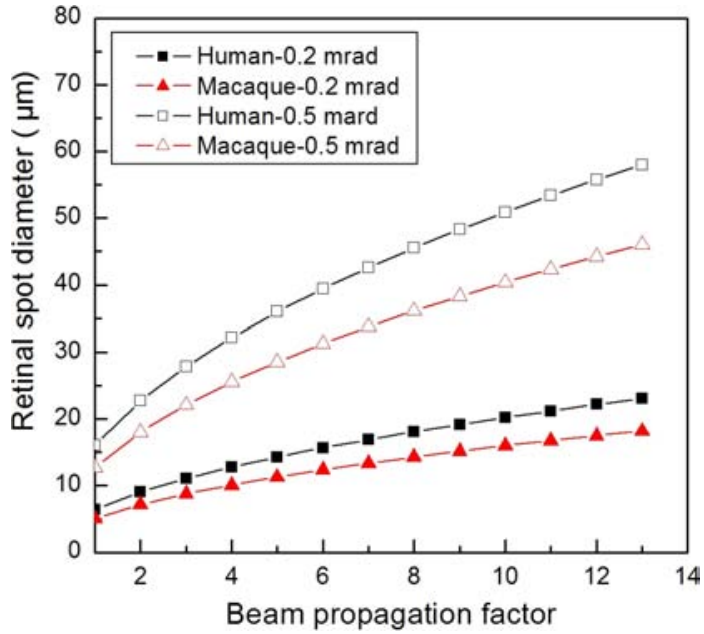

(a)

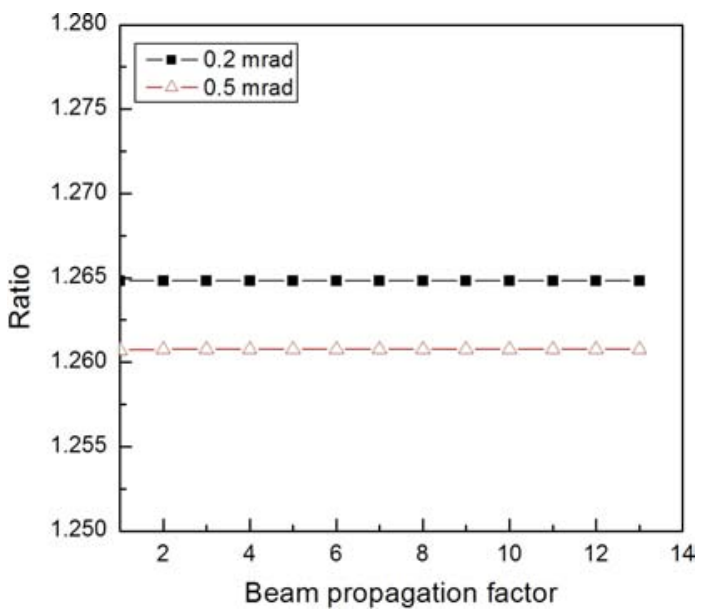

(b)

Fig. 7. Retinal spot diameter versus beam propagation factor for human and macaque (a) and the ratio between the two species (b). Parameters are same with Fig. 5.

macaque, and Fig. 7(b) shows the ratio between them. The laser parameters are same with Fig. 5. We can see that the spot diameter of both species increases monotonously, while the ratio between them is independent of beam propagation factor.

\subsection{Influence of beam divergence on the retinal spot diameter}

Figure 8(a) shows the dependence of the retinal spot diameter of $\mathrm{TEM}_{00}$ and $\mathrm{TEM}_{44}$ mode on beam divergence of the embedded $\mathrm{TEM}_{00}$ mode for human and macaque. The ratio between the retinal

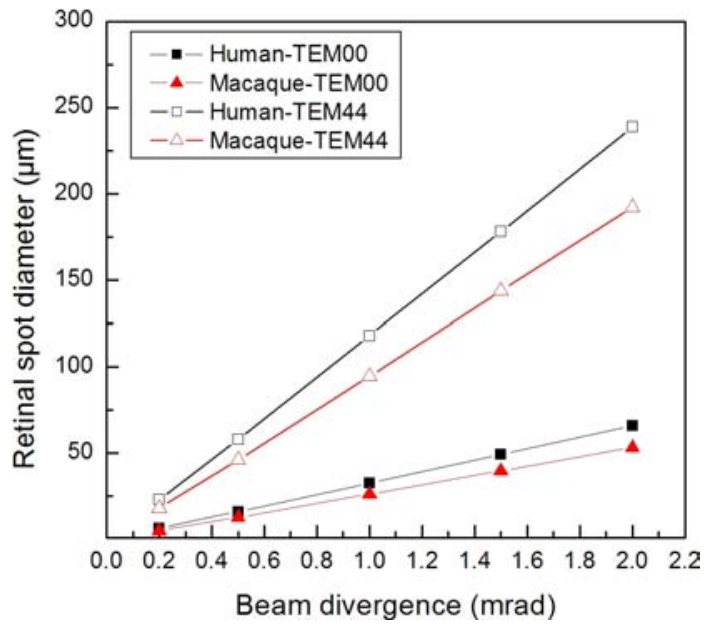

(a) spot diameters of the two species is shown in Fig. 8(b). The laser parameters are same with Fig. 5. We can see that the retinal spot diameter increases rapidly and linearly with beam divergence. The ratio between the retinal spot diameters of the two species decreases slightly with beam divergence.

\section{Discussion}

The aim of this paper was to analyze the influence of laser transverse mode on the retinal spot diameter. To achieve this, an embedded Gaussian model was selected to characterize the propagation of higher

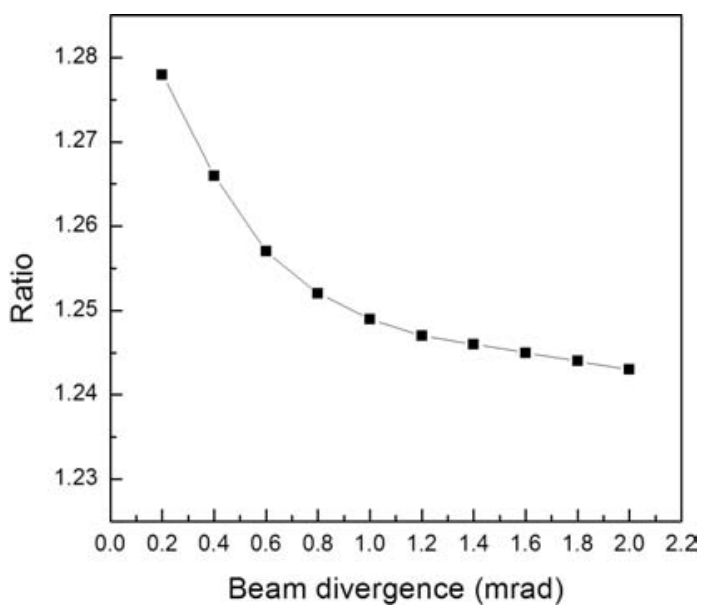

(b)

Fig. 8. Retinal spot diameter of $\mathrm{TEM}_{00}$ and $\mathrm{TEM}_{44}$ mode versus beam divergence of the embedded $\mathrm{TEM}_{00}$ mode for human and macaque (a) and the ratio between the two species (b). 
order mode of Laguerre-Gaussian laser beam; the four-surface schematic eye model was chosen to simulate the real ocular structure of mammal eye. Several assumptions and simplifications were made to facilitate our calculation. Firstly, the eye model was established with spherical surfaces and homogeneous refractive index, which was different from those eye models characterized by aspheric surfaces or gradient refractive index. Secondly, the chromatic aberration has been avoided by setting the retina at the beam waist of the paralleled $\mathrm{TEM}_{00}$ mode for $532 \mathrm{~nm}$. Finally, only the situation of the vertical centered incidence was discussed, while that of optical tilt or decentration was not taken into consideration.

Our calculation indicates that the transverse mode order has remarkable influence on the characteristics of the laser beam transmitted through refractive ocular media. Firstly, as shown in Fig. 5, the beam waist is behind the assumed retina plane for all the calculation cases and its location is independent of the mode order. As the beam divergence increases, the distance between the beam waist and the retina also increases. Secondly, the beam mode order has a significant influence on the retinal spot diameter. From $\mathrm{TEM}_{00}$ to $\mathrm{TEM}_{44}$ mode, the spot diameter increases about 3.6 times for both the 0.2 and $0.5 \mathrm{mrad}$ beam divergences. The contribution of the radial order $p$ on the spot diameter is greater than that of the azimuthal order $l$ (see Fig. 6), while the retinal spot diameter increases with $M^{2}$ factor monotonously, as shown in Fig. 7(a). Thirdly, the increase of retinal spot diameter with radial order $p$, azimuthal order $l$ and $M^{2}$ factor is obvious initially, and then gradually slows down. The beam divergence also has considerable influence on the retinal spot diameter. From Fig. 8(a) we can see that the retinal spot diameter increases rapidly and linearly with beam divergence. From Fig. 7(a) we can see that when the beam divergence is $0.2 \mathrm{mrad}$ or lower, the retinal spot diameter does not exceed $25 \mu \mathrm{m}$ for all the calculated mode orders; while for $0.5 \mathrm{mrad}$, the spot diameter exceeds $25 \mu \mathrm{m}$ for mode orders with $M^{2}$ factor greater than 3 .

It can be inferred from Fig. 1 that the spot size dependence of retinal damage effect can be divided into three distinct regimes. When the retinal spot is larger than $1.7 \mathrm{~mm}$ (100 mrad), the threshold in TIE is proportional to the square of retinal spot diameter; when the retinal spot is smaller than $25 \mu \mathrm{m}(1.5 \mathrm{mrad})$, the threshold is a constant; when the retinal spot is between $25 \mu \mathrm{m}$ and $1.7 \mathrm{~mm}$, the threshold is either linearly or squarely dependent on retinal spot diameter, which is determined by exposure duration. Our calculation shows the retinal spot of higher order modes may either be smaller or larger than $25 \mu \mathrm{m}$, or even larger than $85 \mu \mathrm{m}$ [see Fig. 8(a)]. From above we can infer that the laser mode order has no obvious influence on retinal damage effect when the mode order is low or the beam divergence is small. While for higher mode order or larger beam divergence, the influence should not be neglected and the retinal damage threshold may be elevated. Furthermore, by collimating the incident laser beam, the influence of higher mode order can be reduced. This may be a useful guidance for experimental study.

The macaque monkey is widely used in the study of retinal injury threshold for its similarities with human eye in both the geometrical parameters and physiological function. The retinal injury threshold of macaque monkey is lower than that of human, which has been attributed to lighter pigmentation of human retina. ${ }^{3}$ From our calculation, the retinal spot diameter of macaque monkey is slightly smaller, thus the retinal radiant exposure will be slightly larger than that of human. This makes the MPEs derived from the damage thresholds of macaque safer for human. In addition, the ratio between the retinal spot diameters of human and macaque is independent of mode order (or the beam propagation factor) [see Fig. 7(b)] but slightly depends on beam divergence [see Fig. 8(b)]. So the retinal injury thresholds of the macaque for higher mode order can also be used for the determination of human MPEs, so long as the retinal spot is small enough.

In our analysis, the laser wavelength is $532 \mathrm{~nm}$ and its dispersion effect has been eliminated. It is well known that the retinal spot size of infrared laser beam is much larger than that of visible laser, mainly due to dispersion effect. For example, the retinal spot diameter of $1060 \mathrm{~nm}$ laser for $\mathrm{TEM}_{00}$ mode was about $90-120 \mu \mathrm{m} .{ }^{36-38}$ It can be inferred that compared with the visible light, the influence of the higher order transverse mode of infrared laser on the retinal spot size will be more remarkable. We will concentrate on this problem in subsequent research.

\section{Acknowledgments}

The authors thank the support from the National Nature Science Foundation of China (NSFC), the grant number is 61275194 . 


\section{References}

1. Y. Barkana, M. Belkin, "Laser eye injuries," Surv. Ophthalmaol. 44, 459-478 (2000).

2. B. J. Rockwell, R. J. Thomas, A. Vogel, "Ultrashort laser pulse retinal damage mechanisms and their impact on thresholds," Med. Laser Appl. 25, 84-92 (2010).

3. D. H. Sliney, J. Mellerio, V. P. Gabel, K. Schulmeister, "What is the meaning of thresholds in laser injury experiments? Implications for human exposure limits," Health Phys. 82, 335-347 (2001).

4. J. A. Zuclich, D. J. Lund, P. R. Edsall, R. C. Hollins, P. A. Smith, B. E. Stuck, L. N. Mclin, "Laser induced retinal damage threshold as a function of retinal image size," Proc. SPIE 3591, 335-343 (1999).

5. D. J. Lund, K. Schulmeister, B. Seiser, F. Edthofer, "Laser-induced retinal injury thresholds: Variation with retinal irradiated area," Proc. SPIE 5688, 469-478 (2005).

6. R. L. Vincelette, R. J. Thomas, B. A. Rockwell, C. D. Clerk, A. J. Welch, "First-order model of thermal lensing in a virtual eye," J. Opt. Soc. Am. A 26, 548-558 (2006).

7. C. P. Cain, C. A. Totb, G. D. Noojin, V. Carotbers, D. J. Stolarski, B. J. Rockwell, "Thresholds for visible lesions in the primate eye produced by ultrashort near-infrared laser pulses," Invest. Ophthalmol. Vis. Sci. 40, 2343-2352 (1999).

8. G. Schuele, M. Rumohr, G. Huettmann, R. Brinkmann, "RPE damage thresholds and mechanisms for laser exposure in the microsecond-to-millisecond time regimen," Invest. Ophthalmol. Vis. Sci. 46, 714-719 (2005).

9. C. P. Cain, C. A. Toth, G. D. Noojin, D. J. Stolarski, R. J. Thomas, S. Cora, B. A. Rockwell, "Visible lesion threshold dependence on retina spot size for femtosecond laser pulses," J. Laser Appl. 13, 125-131 (2001).

10. E. S. Beatrice, G. D. Frich, "Retinal laser damage thresholds as a function of the image diameter," Arch. Environ. Health 27, 322-326 (1973).

11. ICBIRP, "ICNIRP guidelines on limits of exposure to laser radiation of wavelengths between $180 \mathrm{~nm}$ and $1000 \mu \mathrm{m}$," Health Phys. 105, 272-295 (2013).

12. K. Schulmeister, B. Seiser, F. Edthofer, D. Lund, "Modelling of the laser spot size dependence of retinal thermal damage," Proc. Int. Laser Safety Conf., pp. 48-57 (2005).

13. K. Schulmeister, B. E. Stuck, D. J. Lund, D. H. Sliney, "Review of thresholds and recommendations for revised exposure limits for laser and optical radiation for thermally induced retinal injury," Health Phys. 100, 210-220 (2011).
14. G. Li, H. Zwick, B. Stuck, D. J. Lund, "On the use of schematic eye models to estimate retinal image quality," J. Biomed. Opt. 5, 307-314 (2000).

15. D. A. Atchision, "Calculating relative retinal image sizes of eyes," Ophthamol. Physiol. Opt. 16, 532-538 (1996).

16. G. D. Frish, "Retinal laser irradiation diameter estimation," Appl. Opt. 11, 939 (1972).

17. R. Navarro, "The optical design of the human eye: A critical review," J. Optom. 2, 3-18 (2009).

18. M. Kong, Z. Gao, L. Chen, X. Li, "Research and development of the optical model of human eye," Laser Technol. 32, 370-373 (2008).

19. J. Einighammer, T. Oltrup, T. Bende, B. Jean, "The individual virtual eye: A computer model for advanced intraocular lens calculation," J. Optom. 2, 70-82 (2009).

20. Y. Chen, C. Jiang, T. Yang, C. Sun, "Development of a human eye model incorporated with intraocular scattering for visual performance assessment," J. Biomed. Opt. 17, 075009 (2012).

21. P. Lapuerta, S. J. Schein, "A four-surface schematic eye of macaque monkey obtained by an optical method," Vision Res. 16, 2245-2254 (1995).

22. A. Hughes, "A schematic eye for the rabbit," Vision Res. 12, 123-138 (1972).

23. A. Hughes, "A schematic eye for the rat," Vision Res. 19, 569-588 (1979).

24. C. Schmucker, F. Schaeffel, "A paraxial schematic eye model for the growing C57BL/6 mouse," Vision Res. 44, 1857-1867 (2004).

25. D. C. O'Shea, Elements of Modern Optical Design, John Wiley \& Sons, New York (1985).

26. J. Alda, Encyclopedia of Optical Engineering, World Scientific Publishing, New York (2003).

27. H. X. Chen, Z. F. Yang, J. R. Wang, P. Chen, H. W. Qian, "A comparative study on ocular damage induced by $1319 \mathrm{~nm}$ laser radiation," Laser in Surgery and Medicine 43, 306-316 (2011).

28. J. P. Taché, "Derivation of ABCD law for LaguerreGaussian beams," Appl. Opt. 26, 2698-2700 (1987).

29. R. Gase, "The multimode laser radiation as a Gaussian shell model beam," J. Mod. Opt. 38(6), 1107-1115 (1991).

30. M. S. Almeida, L. A. Carvalho, "Different schematic eyes and their accuracy to the in vivo eye: A quantitative comparison study," Brazilian J. Phys. 37, 378-387 (2007).

31. R. J. Thomas, R. L. Vincelette, C. D. Clark III, J. Stolarski, L. J. Irvin, G. D. Buffington, "Propagation effects in the assessment of laser damage thresholds to the eye and skin," Proc. SPIE 6435 , 64350A (2007). 
32. D. J. Lund, P. Edsall, B. E. Stuck, "Spectral dependence of retinal thermal injury," Proc. SPIE 3902, 22-34 (2000).

33. U. Grabner, G. Vees, K. Schulmeister, "Beam propagation hazard calculations for telescopic viewing of laser beams," Proc. ILSC 116-125 (2003).

34. D. J. Lund, P. Edsall, "Action spectrum for retinal thermal injury," Proc. SPIE 3591, 324-334 (1999).

35. K. Schulmeister, S. Althaus, U. Grabner, G. Vee, "Beam propagation model for hazard evaluation of Gaussian laser beams," NIR, pp. 793-802 (2004).

36. B. A. Rockwell, D. Hammer, P. K. Kennedy, R. Amnotte, B. Eilert, J. Druessel, D. Payne, S. Phillips,
D. J. Stolarski, G. D. Noojin, R. Thomas, C. P. Cain, "Retinal spot size with wavelength," Proc. SPIE 2975, 148-154 (1997).

37. D. J. Lund, P. Edsall, B. Stuck, "Wavelength dependence of laser-induced retinal injury," Proc. SPIE 5688, 383-393 (2005).

38. R. L. Vincelett, A. J. Welch, R. J. Thomas, B. A. Rockwell, D. J. Lund, "Thermal lensing in ocular media exposed to continuous-wave near-infrared radiation: The 1150-1350 nm region," J. Biomed. Opt. 13, 054005-1-054005-10 (2008). 